\title{
The influence of aging on the effectiveness of heat stress in preventing disuse muscle atrophy
}

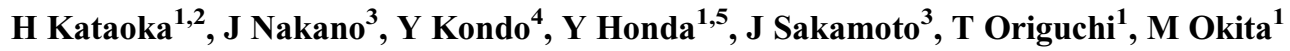 \\ ${ }^{1}$ Department of Locomotive Rehabilitation Science, Unit of Rehabilitation Sciences, Nagasaki University Graduate \\ School of Biomedical Sciences, Nagasaki, Japan \\ ${ }^{2}$ Department of Rehabilitation, Nagasaki Memorial Hospital, Nagasaki, Japan \\ ${ }^{3}$ Unit of Physical Therapy and Occupational Therapy Sciences, Nagasaki University Graduate School of \\ Biomedical Sciences, Nagasaki, Japan \\ ${ }^{4}$ Department of Rehabilitation, Nagasaki Genbaku Hospital, Nagasaki, Japan \\ ${ }^{5}$ Department of Rehabilitation, Nagasaki University Hospital, Nagasaki, Japan
}

Received: March 24, 2016

Accepted: October 30, 2017

\begin{abstract}
This study examined the aging effect on disuse muscle atrophy prevention using heat stress. Wistar rats aged 7 and 60 weeks were divided into three groups as follows: control, immobilized ( $\mathrm{Im})$, and immobilized and heat stressed $(\mathrm{ImH})$. Heat stress was given by immersing the hindlimbs in hot water $\left(42^{\circ} \mathrm{C}\right)$ for 60 min, once in every 3 days and the gastrocnemius (GAS) and soleus (SOL) muscles were extracted after 14 days. Muscle-fiber types were classified using ATPase staining. Heat shock protein 70 (HSP70) was assessed through Western blotting. In GAS muscle of both groups and SOL muscle of 7-week-old rats, the fiber diameter of each muscle type in the ImH group significantly increased compared with that in the Im group. However, this could not be observed in the SOL muscle of the 60-week-old rats. The increased percentage of type-I fibers and variability of types I and II muscle-fiber diameter were evident in the SOL muscle of the 60-week rats. HSP70 was significantly elevated in the ImH group compared with in the Im group in both muscle types of both age groups. Thus, effectiveness of heat stress in the prevention of disuse muscle atrophy appears unsatisfactory in aging muscle fibers.
\end{abstract}

Keywords: aging, disuse muscle atrophy, heat stress, heat shock protein 70, gastrocnemius, soleus

\section{Introduction}

Heat stress reportedly provides protection against disuse muscle atrophy, and heat shock protein 70 (HSP70) induced by heat stress may play an important role in the mechanism underlying this phenomenon $(12,13)$. Naito et al. (12) reported that application of heat stress before an 8-day period of hindlimb unloading in rats elevated HSP70 levels and attenuated muscle atrophy in the soleus (SOL) muscle. We also determined whether heat stress administration during a 7-day period of hindlimb unloading in rats could inhibit progression of muscle atrophy (7). These findings indicated that HSP70 content of both SOL and extensor digitorum longus (EDL) muscles increased in the presence of heat stress; moreover, the diameter of types I and II fibers of SOL and types I and IIA fibers of EDL in heat-stressed rats significantly increased in comparison with unloaded rats without heat stress (7). Selsby and

Corresponding author: Hideki Kataoka, $\mathrm{PhD}$

Department of Locomotive Rehabilitation Science, Unit of Rehabilitation Sciences, Nagasaki University Graduate School of Biomedical Sciences

1-7-1 Sakamoto, Nagasaki-shi 852 8520, Japan

Phone/Fax: +81 95819 7919; E-mail: kataoka-hide@kii.bbiq.jp 
Dodd (16) administered heat stress to rats $24 \mathrm{~h}$ prior to hindlimb immobilization and on alternating days during an 8-day period of immobilization. This research demonstrated higher HSP70 levels and muscle mass of SOL in heat-treated rats relative to immobilized (Im) rats receiving no heat treatment. The results of these studies suggested that heat stress is effective in prevention of disuse muscle atrophy. All reports described above-employed 7- or 8-weekold rats; however, effects of aging on the prevention of disuse muscle atrophy attributable to heat stress remain unclear.

As rats and humans begin to age, decreases in the percentage of type-IIA fibers, increases in the proportion of type-I fibers, and selective atrophy of type-II fibers are evident in skeletal muscles $(4,15)$. Eddinger et al. (4) reported that percentages of types I, IIA, and IIB fibers in rat EDL muscle did not differ from 3 to 30 months of age. However, they also noted that the percentage of type-I fibers of SOL muscle was greater in aged rats in comparison with younger rats. In addition, potential HSP70 expression has been observed in slow skeletal muscles in comparison with fast skeletal muscles (9). In contrast, greater HSP70 expression following heat stress or exercise training has been detected in fast skeletal muscles relative to slow skeletal muscles (11). Therefore, we hypothesized that HSP70 expression as a result of heat stress would differ in connection with aging, and that the preventive effect of heat stress on disuse muscle atrophy differs between aged and young rat skeletal muscles. The objective of this study was to investigate the effect of aging on the preventive effect of heat stress on disuse muscle atrophy in both fast twitch [gastrocnemius (GAS)] and slow twitch (SOL) skeletal muscles in 7- and 60-week-old Wistar rats utilizing histochemical and biochemical analyses.

\section{Materials and Methods}

\section{Animals}

All experiments and procedures were approved by the Ethics Review Committee for Animal Experimentation at Nagasaki University. Rats were kept in cages inside a room with a 12-h dark-light cycle. The temperature and relative humidity of the room were maintained at $25^{\circ} \mathrm{C}$ and $50 \%$, respectively. Food and water were available ad libitum. Eighteen 7-week-old and eighteen 60-week-old male Wistar rats were utilized. Each of the 7-week and 60-week-old rats were randomly divided into one of three experimental groups: (1) sedentary control (Con, $n=6)$; (2) $\operatorname{Im}(n=6)$; and (3) immobilized and heat stress ( $\operatorname{ImH}, n=6)$. Rats in the Im and ImH groups were anesthetized with pentobarbital sodium (40 mg/kg); subsequently, bilateral ankle joints were fixed in full plantar flexion with plaster casts with the triceps surae muscle of the calf immobilized in a shortened position over a 14-day period (1). Rats in Con were anesthetized with pentobarbital sodium $(40 \mathrm{mg} / \mathrm{kg})$ during the time when rats of $\mathrm{Im}$ and $\mathrm{ImH}$ were anesthetized with the same method. The plaster cast, which was positioned from above the knee joint to the distal foot, was changed if it loosened or if hindlimbs of rats displayed swelling. Heat treatment in the ImH group was performed prior to the start of immobilization and then on the 3rd, 6th, 9th, and 12th days during the immobilization period. For heat treatment during the immobilization period, bilateral ankle casts of each rat in the ImH group were removed under pentobarbital sodium anesthesia $(40 \mathrm{mg} / \mathrm{kg})$. Rats in Con and Im were anesthetized with pentobarbital sodium $(40 \mathrm{mg} / \mathrm{kg})$ during the time when rats of $\mathrm{ImH}$ were heated. At the end of the 14-day experimental period, rats were anesthetized with pentobarbital sodium $(40 \mathrm{mg} / \mathrm{kg})$. Subsequently, GAS and SOL 
muscles were quickly removed from the bilateral hindlimbs. Immediately after excision, the wet weight of each muscle was measured and used for subsequent analysis.

\section{Heat stress}

Rats of the ImH group were anesthetized with pentobarbital sodium (40 mg/kg). Subsequently, bilateral hindlimbs of each rat were immersed in hot water at $42{ }^{\circ} \mathrm{C}$ for 60 min once in every 3 days for 2 weeks. We previously established that this method of heat stress significantly increased HSP72 in the GAS and SOL muscles (17).

\section{Histochemical analysis}

The right GAS and SOL muscles were frozen in isopentane cooled to the freezing point with liquid nitrogen. Transverse serial sections $(6 \mu \mathrm{m})$ were cut from the belly of each muscle and collected on glass slides. The sections of both GAS and SOL muscles were stained with hematoxylin-eosin for histological observation. To classify muscle-fiber types, myofibrillar adenosine triphosphatase (myosin ATPase) staining was performed. In this study, the acid preincubation solution was adjusted to $\mathrm{pH} 4.5$ to classify the muscle fibers of the GAS muscle into types I, IIA, and IIB, whereas the alkaline preincubation solution was adjusted to $\mathrm{pH} 10.9$ to classify the muscle fibers of the SOL muscle into types I and II. It is known that the superficial GAS muscle is comprised almost entirely of type-IIB fibers, whereas types I, IIA, and IIB fibers are intermingled in the deep region of the GAS muscle (3). Thus, the deep region of the GAS muscle was assessed in this investigation. Light microscopy images of myosin ATPase staining were obtained using a digital camera (FUJI FILM HC-300Z) at 200× magnification. Subsequently, the muscle-fiber diameters of each fiber type in the GAS and SOL muscles were measured employing the Scion image program for Windows (Scion, Frederick, MD) involving more than 100 fibers. When the total number of the respective fiber types was less than 100, all fibers of that specific type were measured. In addition, the number of fibers of each type in both muscles was counted; subsequently, the proportion of each fiber type was expressed as the percentage of the total number of muscle fibers. The diameter was defined as the maximum diameter across the lesser aspect of the muscle fiber (lesser diameter) (10).

\section{Biochemical analysis}

To determine the HSP70 levels in GAS and SOL muscles, polyacrylamide gel electrophoresis and immunoblotting, as described by Naito et al. (12), were performed. The left GAS in the deep region and the SOL muscles were minced and homogenized in an ice-cold homogenization buffer (10 mM Tris, $10 \mathrm{mM} \mathrm{NaCl}, 0.1 \mathrm{mM}$ EDTA, and $\mathrm{pH}$ 7.6). Homogenates were centrifuged at $12,000 \times g$ for $15 \mathrm{~min}$, after which the supernatants were collected. Following determination of total protein concentrations of the supernatants with a BCA Protein Assay Kit (Thermo Scientific, USA), the concentrations were adjusted to $1 \mathrm{mg} / \mathrm{ml}$. A SDS sample buffer (Bio-Rad, USA) and 2-mercaptoethanol were added to the supernatants, which were then subjected to HSP70 analysis. One-dimensional sodium dodecyl sulfate-polyacrylamide gel electrophoresis (SDS-PAGE) was conducted so as to effect the separation of proteins by molecular weight. The protein from each muscle sample and prestained SDS-PAGE standards (Bio-Rad) were loaded on the $12.5 \%$ polyacrylamide gel. After electrophoretic separation, the proteins were transferred to polyvinylidene difluoride membranes. Subsequently, membranes were blocked for $1 \mathrm{~h}$ with Tris-buffered saline (TBS)/Casein Blocker (Bio-Rad) with $0.05 \%$ Tween 20. The membranes were then incubated for $2 \mathrm{~h}$ with a monoclonal antibody specific for HSP70 (dilution, 1:1000) (Santa Cruz Biotechnology, USA) 
in TBS/Casein Blocker (Bio-Rad) with $0.05 \%$ Tween 20. After washing thrice in TBS with Tween 20 for 5 min each, the blots were incubated with a secondary antibody (goat anti-mouse IgG-conjugated horseradish peroxidase) for $2 \mathrm{~h}$. Upon completion of several washings, the HSP70 bands were detected with a Metal Enhanced DAB Substrate Kit (Pierce). Immunoblots were captured with an image scanner (EPSON GT-X970); quantification of the HSP70 bands was performed with the Scion image program for Windows (Scion, Frederick, MD).

\section{Statistical analysis}

The group differences of muscle-fiber diameters of each fiber type and HSP70 in the GAS and SOL of 7- and 60-week-old rats were analyzed using one-way analysis of variance. Where appropriate, Scheffe's test was performed for post hoc comparisons. In addition, we determined the effect of heat stress by normalizing muscle-fiber diameters of the $\mathrm{ImH}$ rats with respect to the mean muscle-fiber diameter of the Im rats and expressed this value as a percentage. This percentage was then compared between young and aged rats by unpaired $t$-test. $\chi^{2}$ test was used to analyze proportion of each fiber type. Statistical significance was established at $p<0.05$.

\section{Results}

Mean body weight and muscle wet weight

The mean body weight and muscle wet weight of all rats are indicated in Table I. The mean body weight and mean muscle weight were significantly decreased in the Im and $\mathrm{ImH}$ groups compared with the Con group in both 7 - and 60-week-old rats.

\section{Mean muscle-fiber diameter}

The total number of each type of muscle fiber examined is indicated in Table II. In the Im and ImH groups of both the 7- and 60-week-old rats, a decreased muscle-fiber diameter was observed in the GAS and SOL muscles. In the GAS muscle of both the 7- and 60-week-old rats, the mean muscle-fiber diameter of types I, IIA, and IIB fibers in the ImH group significantly increased in comparison with the Im group (Fig. 1 and Table II). Similarly, in the SOL muscle of 7-week-old rats, the mean muscle-fiber diameter of types I and II fibers in the ImH group significantly increased relative to the Im group (Fig. 2 and Table II). In the SOL muscle of 60-week-old rats; however, the mean muscle-fiber diameter of types I and II fibers in the ImH group was indistinct from that of the Im group (Fig. 2 and Table II). Comparison of each muscle-fiber diameter of the ImH group in percentage of the mean and of the Im group between young and aged rats is indicated in Table III. In GAS, the type-I fiber diameter of the ImH group in percentage of the mean and of the Im group is significantly larger in 60-week-old rats compared with 7-week-old rats. However, there are no differences in type IIA and IIB fibers. In SOL, both types I and II fiber diameter of the ImH group in percentage of the mean and of the Im group is significantly smaller in 60 -week-old rats compared with 7-week-old rats.

\section{Percentage distributions of muscle-fiber diameter}

The percentage distributions of the muscle-fiber diameter in the GAS muscle of both 7- and 60-week-old rats are shown in Fig. 3. In each muscle-fiber type of both 7- and 60-week-old rats, the Im and ImH groups shifted to the left in comparison with the Con group; moreover, 
Table I. Data for mean body weight and mean wet weight of soleus (SOL) and gastrocnemius (GAS) muscles in all groups of both ages

\begin{tabular}{|c|c|c|c|}
\hline BW(g) & Con & Im & ImH \\
\hline \multicolumn{4}{|l|}{ 7-week-old rats } \\
\hline Initial & $241.5 \pm 14.6$ & $240.2 \pm 9.8$ & $233.7 \pm 3.7$ \\
\hline Final & $321.3 \pm 24.6$ & $253.7 \pm 11.0^{\mathrm{a}}$ & $242.7 \pm 6.5^{\mathrm{a}}$ \\
\hline \multicolumn{4}{|l|}{ 60-week-old rats } \\
\hline Initial & $918.3 \pm 80.1$ & $802.5 \pm 88.6$ & $808.3 \pm 77.5$ \\
\hline Final & $870.0 \pm 83.6$ & $685.0 \pm 71.0^{\mathrm{a}}$ & $688.3 \pm 74.9^{\mathrm{a}}$ \\
\hline \multicolumn{4}{|c|}{ Wet weight of GAS muscles (mg) } \\
\hline 7-week-old rats & $732 \pm 56.2$ & $424.9 \pm 31.7^{\mathrm{a}}$ & $446.4 \pm 34.8^{\mathrm{a}}$ \\
\hline 60 -week-old rats & $1358.7 \pm 117.9$ & $909.5 \pm 176.5^{\mathrm{a}}$ & $974.2 \pm 174.9^{\mathrm{a}}$ \\
\hline \multicolumn{4}{|c|}{ Wet weight of SOL muscles (mg) } \\
\hline 7-week-old rats & $129.7 \pm 14.7$ & $51.9 \pm 3.7^{\mathrm{a}}$ & $56.5 \pm 3.7^{\mathrm{a}}$ \\
\hline 60 -week-old rats & $265.1 \pm 31.0$ & $176.2 \pm 31.1^{\mathrm{a}}$ & $164.6 \pm 15.6^{\mathrm{a}}$ \\
\hline
\end{tabular}

BW: body weight; Con: sedentary control; Im: immobilized; ImH: immobilized and heat stressed.

${ }^{a}$ Significantly different from Con group $(p<0.05)$

the ImH group was located to the right relative to the Im group. The percentage distributions of muscle-fiber diameter in the SOL muscle of both 7- and 60-week-old rats appear in Fig. 4. Results of each muscle-fiber type in 7-week-old rats were similar to those of the GAS muscle. In all groups of 60-week-old rats, however, the variability of diameter of types I and II muscle fibers increased. This tendency was notably accepted in type-II fibers. Furthermore, each muscle fiber type of the Im and ImH groups shifted to the left in comparison with the Con group.

\section{Proportions of muscle fiber types}

The means \pm SD with respect to percentages of types I, IIA, and IIB fibers in the GAS muscle of 7-week-old rats were $23.4 \% \pm 5.0 \%, 23.7 \% \pm 4.2 \%$, and $52.9 \% \pm 5.4 \%$ in the Con group, $22.5 \% \pm 2.3 \%, 19.8 \% \pm 2.0 \%$, and $57.7 \% \pm 2.2 \%$ in the Im group, and $23.6 \pm 3.7 \%$, $22.4 \pm 2.1 \%$, and $54.0 \pm 5.3 \%$ in the $\mathrm{ImH}$ group, respectively. No difference in musclefiber-type composition was observed across the three groups. Similarly, muscle-fiber-type composition in the GAS muscle of 60 -week-old rats exhibited no difference across the three groups. In addition, no difference in muscle-fiber-type composition was evident between the corresponding groups of the 7- and 60-week-old rats (Table II). The means \pm SD for the percentages of types I and II fibers in the SOL muscle of 7-week-old rats were $77.1 \% \pm 5.3 \%$ and $22.9 \% \pm 5.3 \%$ in the Con group, $71.8 \% \pm 8.4 \%$ and $28.2 \% \pm 8.4 \%$ in the Im group, and $73.8 \% \pm 9.7 \%$ and $26.2 \% \pm 9.7 \%$ in the ImH group, respectively. No difference in musclefiber-type composition was detected across the three groups. The means \pm SD for percentages of types I and II fibers in the SOL muscle of 60 -week-old rats were $92.6 \% \pm 5.9 \%$ and $7.4 \% \pm 5.9 \%$ in the Con group, $95.6 \% \pm 4.5 \%$ and $4.4 \% \pm 4.5 \%$ in the Im group, and 
Table II. Data for mean muscle-fiber diameter of and total number of fibers in soleus (SOL) and gastrocnemius (GAS) muscles in all groups

\begin{tabular}{|c|c|c|c|c|}
\hline & \multirow[b]{2}{*}{ Muscle-fiber types } & \multicolumn{3}{|c|}{$\begin{array}{l}\text { Mean muscle-fiber diameter }(\mu \mathrm{m}) / \text { total number of fibers } \\
\text { examined }\end{array}$} \\
\hline & & Con & Im & $\operatorname{ImH}$ \\
\hline \multicolumn{5}{|l|}{$G A S$} \\
\hline \multirow[t]{3}{*}{ 7-week-old rats } & Type I & $37.5 \pm 7.7 / 777$ & $26.8 \pm 5.7^{\mathrm{a} / 879}$ & $28.6 \pm 5.7^{\mathrm{a}, \mathrm{b}} / 739$ \\
\hline & Type IIA & $37.1 \pm 6.1 / 777$ & $27.9 \pm 4.7^{\mathrm{a}} / 687$ & $31.1 \pm 5.3^{\mathrm{a}, \mathrm{b}} / 752$ \\
\hline & Type IIB & $42.7 \pm 8.2 / 833$ & $33.0 \pm 7.0 \mathrm{a} / 1184$ & $34.9 \pm 7.7^{\mathrm{a}, \mathrm{b}} / 927$ \\
\hline \multirow[t]{3}{*}{60 -week-old rats } & Type I & $45.5 \pm 9.0 / 708$ & $36.7 \pm 7.0^{\mathrm{a}} / 661$ & $42.1 \pm 8.0^{\mathrm{a}, \mathrm{b}} / 738$ \\
\hline & Type IIA & $48.3 \pm 8.7 / 661$ & $39.9 \pm 7.5^{\mathrm{a}} / 753$ & $44.4 \pm 7.9^{\mathrm{a}, \mathrm{b}} / 694$ \\
\hline & Type IIB & $54.5 \pm 11.6 / 813$ & $44.1 \pm 9.4^{\mathrm{a} / 776}$ & $46.3 \pm 10.3^{\mathrm{a}, \mathrm{b}} / 857$ \\
\hline \multicolumn{5}{|l|}{$S O L$} \\
\hline \multirow[t]{2}{*}{ 7-week-old rats } & Type I & $47.3 \pm 8.3 / 798$ & $31.5 \pm 6.6^{\mathrm{a}} / 1272$ & $35.2 \pm 6.2^{\mathrm{a}, \mathrm{b}} / 1145$ \\
\hline & Type II & $43.1 \pm 8.6 / 688$ & $25.5 \pm 5.5^{\mathrm{a}} / 801$ & $32.4 \pm 6.4^{\mathrm{a}, \mathrm{b}} / 717$ \\
\hline \multirow[t]{2}{*}{60 -week-old rats } & Type I & $54.0 \pm 10.2 / 775$ & $44.2 \pm 9.5^{\mathrm{a}} / 833$ & $45.1 \pm 9.2^{\mathrm{a} / 935}$ \\
\hline & Type II & $48.9 \pm 13.7 / 476$ & $39.4 \pm 9.5^{\mathrm{a}} / 477$ & $38.5 \pm 10.9^{\mathrm{a}} / 336$ \\
\hline
\end{tabular}

Values are mean \pm SD. Con: sedentary control; Im: immobilized; ImH: immobilized and heat stressed.

${ }^{a}$ Significantly different from Con group $(p<0.05)$.

${ }^{\mathrm{b}}$ Significantly different from Im group $(p<0.05)$

A

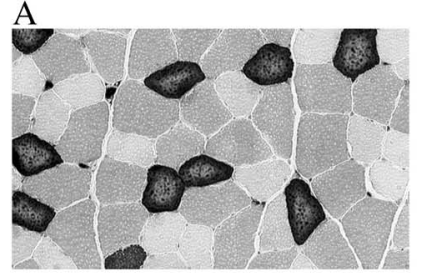

D

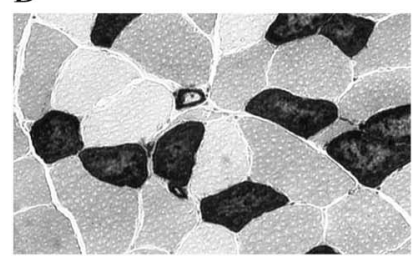

B

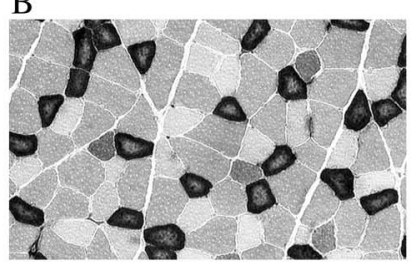

E

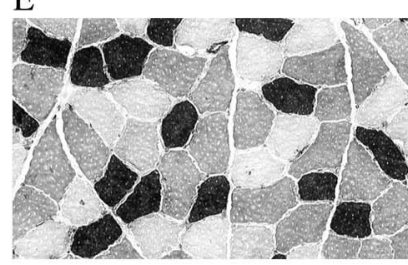

C

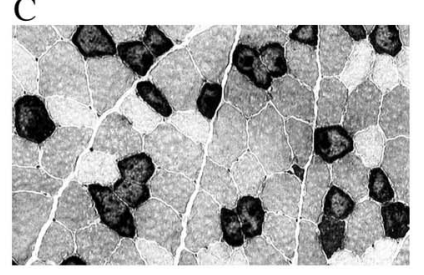

F

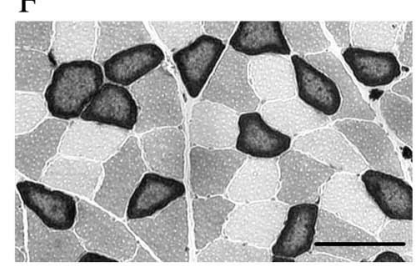

Fig. 1. Light micrographs of histological sections of gastrocnemius muscles stained for myosin-ATPase (pH 4.5). Type-I fibers display dark stain; type-IIA fibers exhibit light stain; type-IIB fibers demonstrate intermediate stain. Upper row represents 7-week-old rats (A: Con, B: Im, and C: $\operatorname{ImH}$ ); lower row represents 60-week-old rats (D: Con, E: Im, and F: $\operatorname{ImH})$. Bar $=100 \mu \mathrm{m}$ 

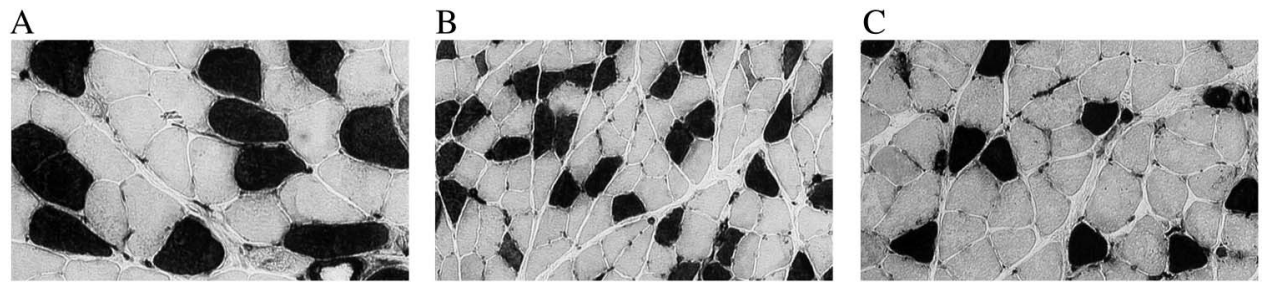

\section{$\mathrm{D}$}
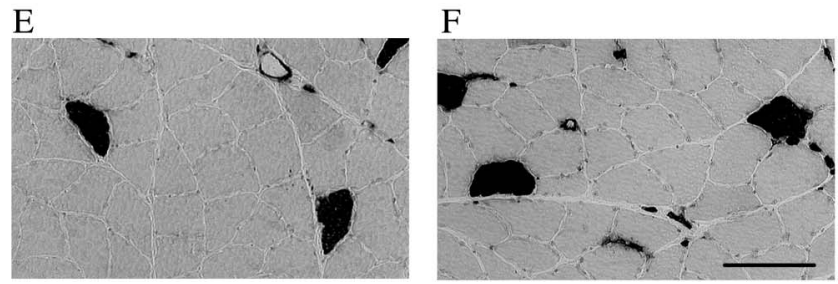

Fig. 2. Light micrographs of histological sections of soleus muscles stained for myosin-ATPase (pH 10.5). Type-I fibers display light stain; type-II fibers exhibit dark stain. Upper row represents 7-week-old rats (A: Con, B: Im, and $\mathrm{C}: \operatorname{ImH}$ ); lower row represents 60-week-old rats (D: Con, E: Im, and F: $\operatorname{ImH}) . \mathrm{Bar}=100 \mu \mathrm{m}$

Table III. Comparison of each muscle-fiber diameter of $\mathrm{ImH}$ in percentage of the mean and of Im between young and aged rats

\begin{tabular}{|l|c|c|c|}
\hline & & $\mathbf{7}$ weeks & $\mathbf{6 0}$ weeks \\
\hline \multirow{3}{*}{ GAS } & Type I & $106.8 \pm 21.4$ & $114.7 \pm 21.7^{\mathrm{a}}$ \\
\cline { 2 - 4 } & Type IIA & $111.4 \pm 19.1$ & $111.3 \pm 11.9$ \\
\cline { 2 - 4 } & Type IIB & $105.3 \pm 23.4$ & $105.1 \pm 23.4$ \\
\hline \multirow{2}{*}{ SOL } & Type I & $111.6 \pm 19.6$ & $101.6 \pm 21.2^{\mathrm{a}}$ \\
\cline { 2 - 4 } & Type II & $127.1 \pm 5.0$ & $86.6 \pm 24.4^{\mathrm{a}}$ \\
\hline
\end{tabular}

GS: gastrocnemius; SOL: soleus.

${ }^{\text {a }}$ Significantly different from 7-week-old rats $(p<0.05)$

$96.4 \% \pm 3.2 \%$ and $3.6 \% \pm 3.2 \%$ in the ImH group, respectively. Similarly, in 7-week-old rats, no difference in the muscle-fiber-type composition was evident across the three groups. However, comparison between the corresponding groups of 7- and 60-week-old rats revealed that all groups of 60-week-old rats displayed an increase in the percentage of type-I fibers and a decrease in the percentage of type-II fibers (Table IV).

\section{HSP70 content}

In the GAS muscle, the result for HSP70 content was identical in 7- and 60-week-old rats. No significant differences were observed between the Con and Im groups. HSP70 expression in the ImH group was significantly higher than that in the other groups (Fig. 5). In the SOL muscle, the result of HSP70 content was identical in 7- and 60-week-old rats. The Im group displayed a significant decrease relative to the Con group, whereas the $\mathrm{ImH}$ group exhibited a significant increase in comparison with the Im group. No significant difference was detected between the Con and ImH groups (Fig. 6). 

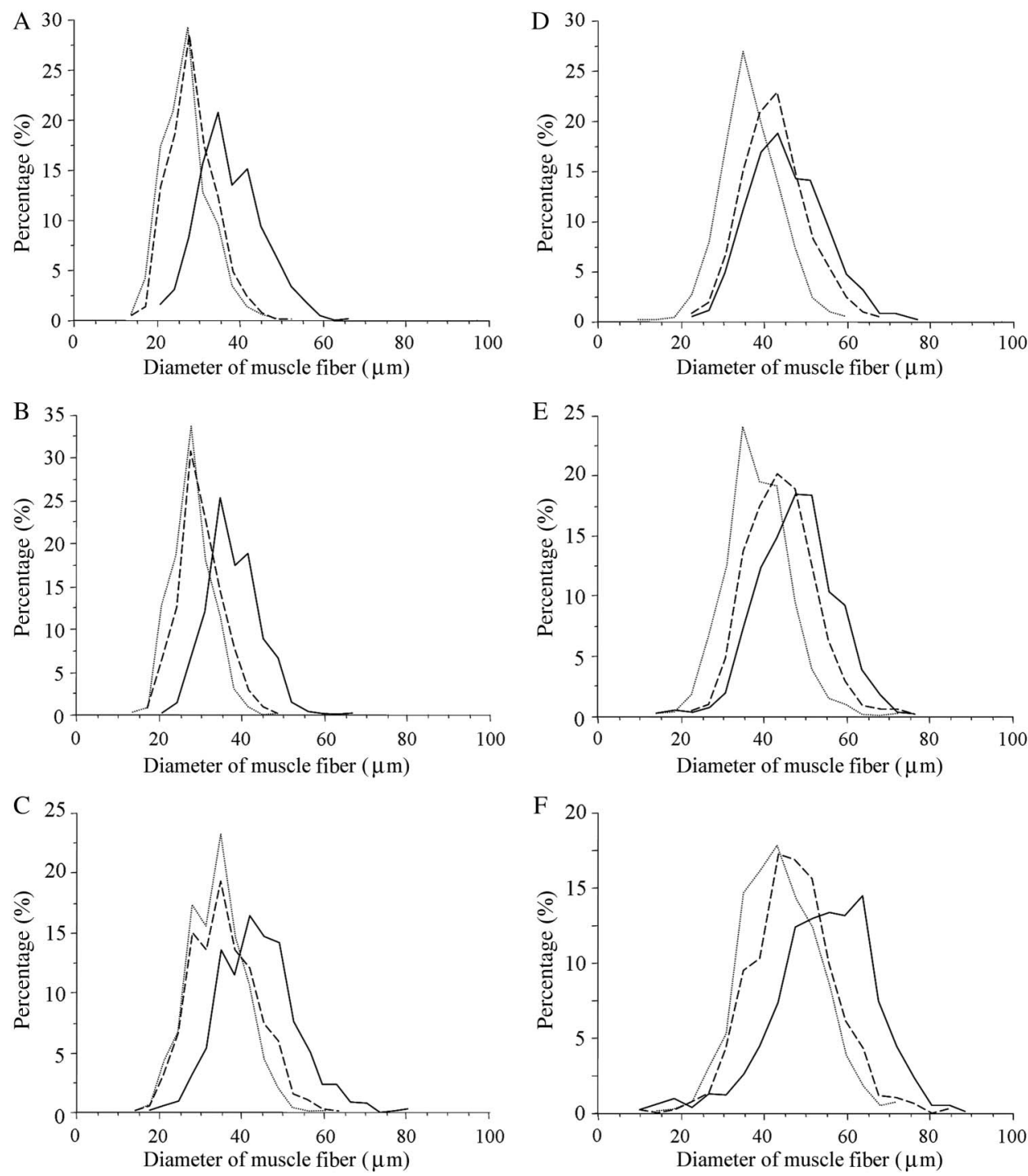

Fig. 3. Percentage distributions of muscle-fiber diameter of each muscle-fiber type in gastrocnemius muscles. In 7-week-old rats (left column), each muscle-fiber type (A: type I, B: type IIA, and C: type IIB) in the Im (dotted line) and $\mathrm{ImH}$ (broken line) groups shifted to the left in comparison with the Con (solid line) group. The ImH group was located to the right relative to the Im group. Similarly, in 60-week-old rats, each muscle-fiber type (right column; D: type I, E: type IIA, and F: type IIB) in the Im (dotted line) and ImH (broken line) groups shifted to the left in comparison with the Con (solid line) group. The ImH group was located to the right relative to the Im group

\section{Discussion}

The main finding of this study is that heat stress inhibited disuse atrophy of the GAS muscle of 7- and 60-week-old rats and the SOL muscle of 7-week-old rats, but not in the SOL muscle of 60-week-old rats. The effect of heat stress on prevention of disuse muscle atrophy appears unsatisfactory for the muscles, which include fibers changed by aging. 

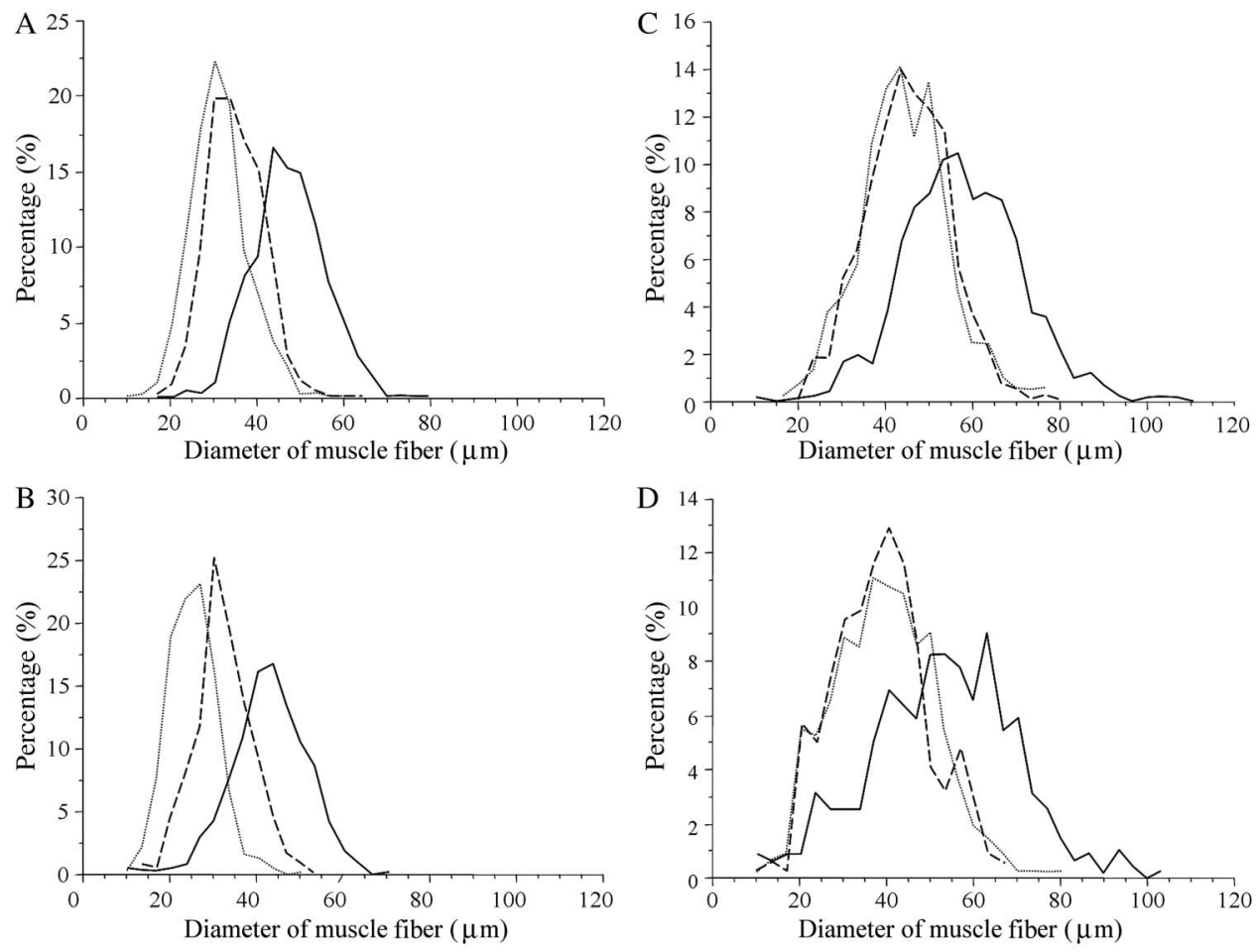

Fig. 4. Percentage distributions of muscle-fiber diameter of each muscle-fiber type in soleus muscles. In 7-week-old rats (left column), each muscle-fiber type (A: type I and B: type II) in the Im (dotted line) and ImH (broken line) groups shifted to the left in comparison with the Con (solid line) group. The ImH group was located to the right relative to the Im group. In 60-week-old rats in all groups (right column; C: type I and D: type II), however, a large increase in the variability of the types I and II muscle-fiber diameters was evident; moreover, each muscle-fiber type in the Im (dotted line) and $\operatorname{ImH}$ (broken line) groups shifted to the left in comparison with the Con (solid line) group. The peak in percentage distributions of muscle-fiber diameter of the ImH group did not differ from that of the Im group

HSP70 content was significantly greater in the ImH group in comparison with the Con and Im groups; thus, it is apparent that HSP70 in the GAS muscle of 7- and 60-week-old rats was induced by heat stress involving a hot bath set at $42^{\circ} \mathrm{C}$. The HSP70 content in the SOL muscle of 7- and 60-week-old rats was significantly lower in the Im group in comparison with the Con group; moreover, no difference was evident between the $\mathrm{CON}$ and $\mathrm{ImH}$ groups. Heat stress may maintain HSP70 contents declining with immobilization. Thus, HSP70 would be expressed in the SOL muscles in both age classes as a result of heat stress. Locke (9) demonstrated HSP72 (the inducible member of the HSP70 family) expression in both SOL and white GAS muscles of 20-month-old rats induced by heat stress. The diminished ability to induce HSPs in aged cells and tissues appears to result from decreased heat shock factor (HSF) activation (9). Locke (9) demonstrated that after heat shock, both fast- and slow-twitch skeletal muscles from aged rats demonstrated similar HSF activation and HSP72 accumulation as the same muscles from adult rats. Although we have not examined HSF activation, the findings of our study and the report of Locke (9) suggested that expression of HSP70 in skeletal muscles following heat stress is not affected by aging. 
Table IV. Proportions of muscle fiber types of soleus (SOL) and gastrocnemius (GAS) muscles in all groups

\begin{tabular}{|c|c|c|c|c|}
\hline & \multirow[b]{2}{*}{ Muscle-fiber types } & \multicolumn{3}{|c|}{ Percentage of each muscle fiber type (\%) } \\
\hline & & Con & Im & $\mathbf{I m H}$ \\
\hline \multicolumn{5}{|l|}{ GAS } \\
\hline \multirow[t]{3}{*}{ 7-week-old rats } & Type I & $23.4 \pm 5.0$ & $22.5 \pm 2.3$ & $23.6 \pm 3.7$ \\
\hline & Type IIA & $23.7 \pm 4.2$ & $19.8 \pm 2.0$ & $22.4 \pm 2.1$ \\
\hline & Type IIB & $52.9 \pm 5.4$ & $57.7 \pm 2.2$ & $54.0 \pm 5.3$ \\
\hline \multirow[t]{3}{*}{60 -week-old rats } & Type I & $20.3 \pm 3.6$ & $19.0 \pm 1.8$ & $21.2 \pm 6.1$ \\
\hline & Type IIA & $22.3 \pm 5.0$ & $25.1 \pm 3.0$ & $18.8 \pm 3.6$ \\
\hline & Type IIB & $57.4 \pm 6.9$ & $55.9 \pm 3.9$ & $60.0 \pm 8.7$ \\
\hline \multicolumn{5}{|l|}{$S O L$} \\
\hline \multirow[t]{2}{*}{ 7-week-old rats } & Type I & $77.1 \pm 5.3$ & $71.8 \pm 8.4$ & $73.8 \pm 9.7$ \\
\hline & Type II & $22.9 \pm 5.3$ & $28.2 \pm 8.4$ & $26.2 \pm 9.7$ \\
\hline \multirow[t]{2}{*}{60 -week-old rats } & Type I & $92.6 \pm 5.9^{\mathrm{a}}$ & $95.6 \pm 4.5^{\mathrm{a}}$ & $96.4 \pm 3.2^{\mathrm{a}}$ \\
\hline & Type II & $7.4 \pm 5.9^{\mathrm{a}}$ & $4.4 \pm 4.5^{\mathrm{a}}$ & $3.6 \pm 3.2^{\mathrm{a}}$ \\
\hline
\end{tabular}

Values are means \pm SD.

${ }^{a}$ Significantly different from the same group of 7-week-old rats $(p<0.05)$

In both age groups, HSP70 expression induced by heat stress was greater in the GAS muscle than in the SOL muscle. The differences in terms of muscle-fiber-type composition in the GAS and the SOL muscles would influence these results. Briefly, in previous studies $(9,12-14)$, higher HSP70 expression after heat stress or exercise training was detected in fast skeletal muscles in comparison with slow skeletal muscles. Potential HSP70 expression in the GAS muscle is lower than that in the SOL muscle; consequently, the GAS muscle must induce greater HSP70 expression levels against heat stress relative to the SOL muscle.

Based on the findings of the Im and ImH groups in 7-week-old rats, the effects of heat stress on the prevention of disuse skeletal muscle atrophy were detectable in the GAS and SOL muscles. This mechanism would be related to HSP70 expression in myocytes (5). HSP70 can play important roles in chaperoning nascent peptides during elongation and folding, and in the repair of damaged proteins (12). In addition, we previously reported that heat stress maintained the level of atrogene expression, increased Hsp72 and inhibited glucocorticoid-induced myopathy (10). Kondo et al. (8) reported that oxidative stress is an important mechanism of skeletal muscle atrophy by immobilization. Furthermore, the study of Selsby and Dodd (16) indicated that heat stress could reduce oxidative stress of rat SOL muscle during immobilization. They also hypothesized that HSP70 may play a role in protection against oxidative stress.

Based on the results of the Im and ImH groups in 60w rats, the effects of heat stress on the prevention of disuse skeletal muscle atrophy were detectable in the GAS but not in the SOL muscle. Thus, differential effects of heat stress on prevention of disuse skeletal muscle atrophy in fast (GAS) and slow (SOL) muscles were observed. In addition, our findings 
A

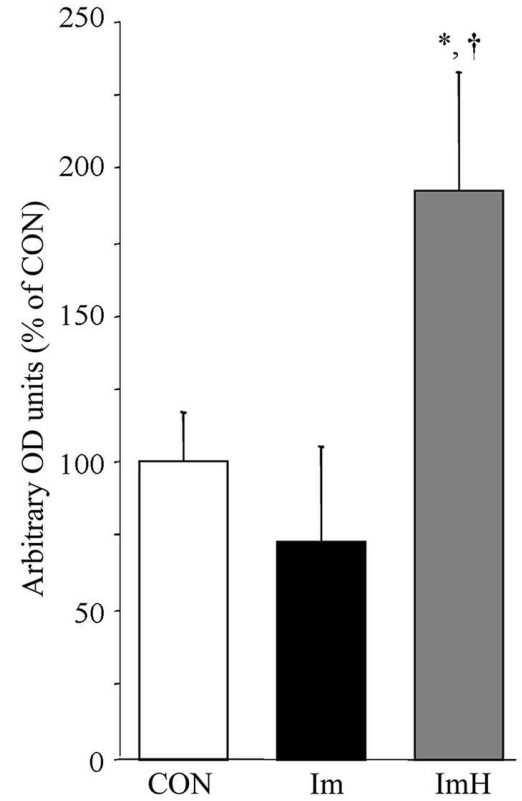

B

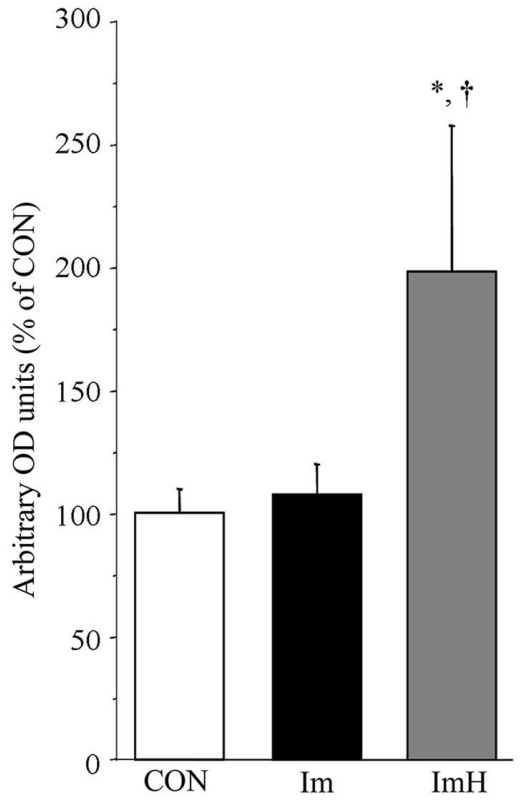

Fig. 5. HSP70 representative blot and relative expression in gastrocnemius muscles of 7- (A) and 60-week-old (B) rats. In both 7- and 60-week-old rats, no significant difference was observed between the Con and Im groups. HSP70 expression in the ImH group was significantly higher than that in the other groups. Data are expressed as mean \pm SD.

*Significantly different from CON group $(p<0.05)$. ${ }^{\dagger}$ Significantly different from $\operatorname{Im}$ group $(p<0.05)$

demonstrated that changes in muscle-fiber-type composition and the percentage distribution of muscle-fiber diameters due to aging differed between the GAS and SOL muscles.

There were no differences in muscle-fiber-type composition and the percentage distributions of muscle-fiber diameters between 60- and 7-week-old rats in the GAS muscle. On the other hand, the increase in type-I fibers and the variability of percentage distributions of fiber diameter were detected in the SOL muscle of 60 -week-old rats. The mechanism of change in fiber-type composition because of aging may be produced by motor unit remodeling. Namely, selective denervation of type-II fibers occurred; moreover, the type-II fibers may be reinnervated by sprouting of nerves from type-I motor units (2). Husom et al. (6) reported a decline in type-I skeletal muscle-fiber diameter in the SOL muscle but not in the GAS muscle in 6- to 40-month-old rats. Thus, it is obvious that the SOL muscle is more susceptible to aging than the GAS muscle. In this study, changes due to aging in the percentage distribution of muscle-fiber diameter in the SOL muscle may be indicated with respect to denervation of type-II fibers. Furthermore, differential effects of heat stress on the prevention of disuse skeletal muscle atrophy in fast (GAS) and slow (SOL) muscles would have occurred by these differences in age-related changes.

As clinically implicated, heat stress can be used to prevent disuse skeletal muscle atrophy for not only young but also for older people. However, the effects may be different between fast or slow muscles in older people.

In conclusion, the current findings suggested that heat stress would influence the prevention of disuse muscle atrophy in fast muscles characterized by little neurogenic 
A
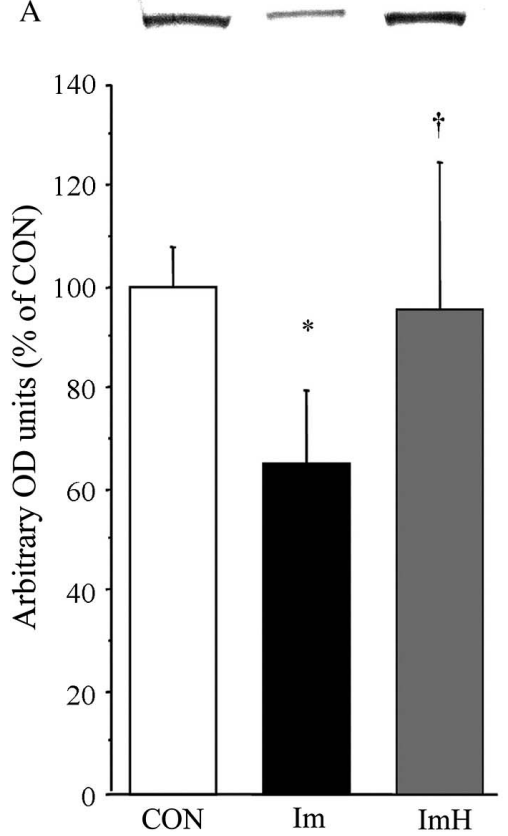

B

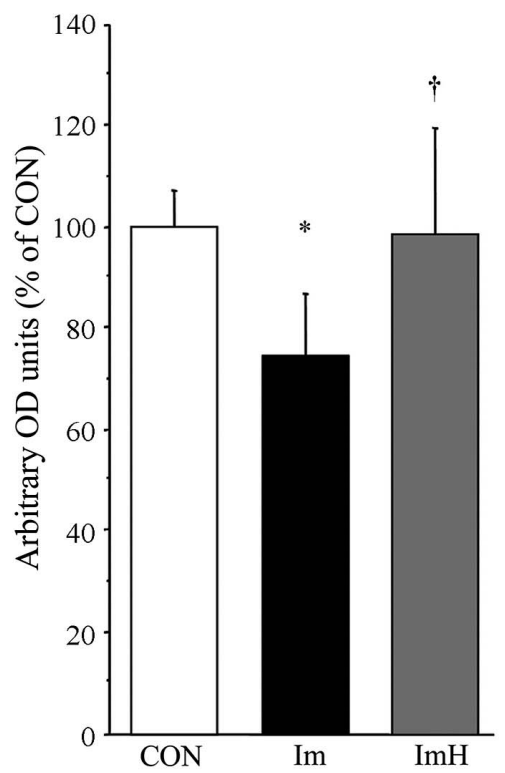

Fig. 6. HSP70 representative blot and relative expression in soleus muscles of 7- (A) and 60-week-old (B) rats. In both 7- and 60-week-old rats, HSP70 expression in the Im group was significantly lower than that in the Con group, whereas expression in the ImH group was significantly higher than that in the Im group. However, no significant difference was observed between the Con and ImH groups. Data are expressed as mean \pm SD.

*Significantly different from Con group $(p<0.05)$. ${ }^{\dagger}$ Significantly different from $\operatorname{Im}$ group $(p<0.05)$

change in myofibers because of aging. Moreover, we hypothesized that HSP70 induced by heat stress may be related to this mechanism. In contrast, although heat stress induced the expression of HSP70 in aged slow muscle, as aged slow muscles contained many myofibers displaying neurogenic changes, such as denervation and/or reinnervation, progression of disuse muscle fiber atrophy was not reduced by heat stress. Additional studies are necessary to clarify this phenomenon.

\section{Acknowledgements}

The authors would like to thank Toshiro Yoshimura of the Department of Locomotive Rehabilitation Science, Unit of Rehabilitation Sciences, Nagasaki University Graduate School of Biomedical Sciences, for his support in the histochemical analysis.

\section{REFERENCES}

1. Ansved T: Effects of immobilization on the rat soleus muscle in relation to age. Acta Physiol. Scand. 154, 291-302 (1995)

2. Brooks SV, Faulkner JA: Skeletal muscle weakness in old age: underlying mechanisms. Med. Sci. Sports. Exerc. 26, 432-439 (1994)

3. Delp MD, Duan C: Composition and size of type I, IIA, IID/X, and IIB fibers and citrate synthase activity of rat muscle. J. Appl. Physiol. 80, 261-270 (1996) 
4. Eddinger TJ, Moss RL, Cassens RG: Fiber number and type composition extensor digitorum longus, soleus, and diaphragm muscles with aging in Fisher 344 rats. J. Histochem. Cytochem. 33, 1033-1041 (1985)

5. Goto K, Okuyama R, Sugiyama H, Honda M, Kobayashi T, Uehara K, Akema T, Sugiura T, Yamada S, Ohira Y, Yoshioka T: Effects of heat stress and mechanical stretch on protein expression in cultured skeletal muscle cells. Pflugers. Arch. 447, 247-253 (2003)

6. Husom AD, Ferrington DA, Thompson LV: Age-related differences in the adaptive potential of type I skeletal muscle fibers. Exp. Gerontol. 40, 227-235 (2005)

7. Kataoka H, Okita M, Nakai K, Toyoda N, Yoshikawa S, Watanabe Y, Nakano J: Effects of heat stress on prevention of disuse muscle atrophy in rat skeletal muscle. Rigakuryohougaku 31, 331-336 (2004)

8. Kondo H, Nakagaki I, Sasaki S, Hori S, Itokawa Y: Mechanism of oxidative stress in skeletal muscle atrophied by immobilization. Am. J. Physiol. 265, E839-E844 (1993)

9. Locke M: Heat shock transcription factor activation and Hsp72 accumulation in aged skeletal muscle. Cell Stress Chaperones 5, 45-51 (2000)

10. Morimoto Y, Kondo Y, Kataoka H, Honda Y, Kozu R, Sakamoto J, Nakano J, Origuchi T, Yoshimura T, Okita M: Heat treatment inhibits skeletal muscle atrophy of glucocorticoid-induced myopathy in rats. Physiol. Res. 64(6), 897-905 (2015)

11. Naito H, Powers SK, Demirel HA, Aoki J: Exercise training increases heat shock protein in skeletal muscle of old rats. Med. Sci. Sports Exerc. 33, 729-734 (2001)

12. Naito H, Powers SK, Demirel HA, Sugiura T, Dodd SL, Aoki J: Heat stress attenuates skeletal muscle atrophy in hindlimb-unweighted rats. J. Appl. Physiol. 88, 359-363 (2000)

13. Oishi Y, Taniguchi K, Matsumoto H, Ishihara A, Ohira Y, Roy RR: Differential response of HSPs to heat stress in slow and fast regions of rat gastrocnemius muscle. Muscle Nerve 28, 587-594 (2003)

14. Oishi Y, Taniguchi K, Matsumoto H, Ishihara A, Ohira Y, Roy RR: Muscle type-specific response of HSP60, HSP72, and HSC73 during recovery after elevation of muscle temperature. J. Appl. Physiol. 92, 1097-1103 (2002)

15. Scelsi R, Marchetti C, Poggi P: Histochemical and ultrastructural aspects of M. vastus lateralis in sedentary old people (age 65-89 years). Acta Neuropathol. 51, 99-105 (1980)

16. Selsby JT, Dodd SL: Heat treatment reduces oxidative stress and protects muscle mass during immobilization. Am. J. Physiol. Regul. Integr. Comp. Physiol. 289, R134-R139 (2005)

17. Yoshida N, Morimoto Y, Kataoka H, Sakamoto J, Nakano J, Okita M: Effects of combination therapy of heat stress and muscle contraction exercise induced by neuromuscular electrical stimulation on disuse atrophy in the gastrocnemius. J. Phys. Ther. Sci. 25, 201-206 (2013) 